\title{
Una visión actual de la diversidad y distribución de los pinos de México
}

\section{Diversity and distribution of Mexican pines, an overview}

\author{
Arturo Sánchez-González ${ }^{1}$
}

\section{RESUMEN}

\begin{abstract}
México es considerado un centro secundario de diversidad del género Pinus. Un análisis de la literatura más reciente indica que los pinos migraron hacia el actual territorio mexicano desde el hemisferio norte a través de corredores naturales e impulsados por los cambios climáticos del pasado. Los procesos de diversificación del género ocurrieron en las principales cadenas montañosas de México, que funcionaron como corredores biológicos o como islas biogeográficas que definieron el patrón de distribución general actual de los pinos en cinco grandes regiones. Recientemente se reconocen para México 46 especies, 3 subespecies y 22 variedades de pinos. El $55 \%$ de estos taxa son endémicos. Lo que nos convierte en la nación con mayor diversidad al contar con alrededor de $42 \%$ de las especies del mundo. Los pinos mexicanos son uno de los recursos naturales más valiosos por su uso tradicional y comercial, por su importancia cultural y por los servicios ambientales que ofrecen. A pesar de ello la continua disminución de la cobertura de los bosques de pino y pino-encino, por causa de las actividades humanas y por los incendios forestales, es alarmante. Al menos 20 taxa de pinos mexicanos están en alguna categoría de riesgo y dos de ellos: Pinus maximartinezii y $P$. muri cata se encuentran en peligro de extinción. Los programas nacionales e internacionales para el uso sostenible y la conservación del género Pinus en México aún no son eficientes y suficientes.
\end{abstract}

PALABRAS CLAVE:

Distribución, diversidad, ecología, endemismos, gimnospermas, Pinus.

\section{ABSTRACT}

Mexico is considered a secondary center of diversity of the genus Pinus. Published literature indicates that this genus migrated from the north hemisphere through down natural corridors to the actual Mexican territory, forced by past climatic changes. The diversification of the genus happened on main mountain chains and those acted like biogeographical islands or biological corridors, modeling the actual distribution patterns of the Pinus in five mayor regions. Recently, forty six species, three subspecies and twenty two varieties from pines are recognized to Mexico. At least $55 \%$ from those species are endemic). Because of that, is the biggest diverse country of pine species from the world, with $42 \%$ of them. Mexican pines are one of the most valuable natural resources because of their traditional and commercial relevance and the environmental services that they provide. In spite of the national and regional significance, the continuous decreasing of covering areas from pine and pineoak forests because of the human activities and fire forest is alarming. At least 20 Mexican taxa are in some risk status and two of them: Pinus maximartinezii and $P$. muricata are classified like endangered species. National and international programs for sustainable use and conservation from Pinus are not efficient and sufficient yet.

KEY WORDS:

Distribution, diversity, ecology, endemism, gymnosperm, Pinus. 


\section{INTRODUCCIÓN}

Las gimnospermas comprenden alrededor de 900 especies, la mayoría localizadas en el hemisferio norte, alrededor de $60 \%$ son coníferas (Farjon, 2003; Earle, 2007). El registro fósil muestra que los ancestros de la familia Pinaceae evolucionaron hacia finales del Carbonífero, hace aproximadamente 300 millones de años (Hilton et al., 2003) y que el género Pinus comenzó su diversificación en el Cretácico inferior (Miller, 1999).

La riqueza aproximada de especies de pinos a nivel mundial es de 111 especies (Price et al., 1998). En México y América Central se localizan alrededor de 46 especies de pinos, con diversas variedades y formas (Perry et al., 1998). México es un centro secundario de diversidad de Pinus al contar con cerca de $42 \%$ de las especies y un alto porcentaje de endemismos (>55\%) concentrados en islas biogeográficas. Tal es el caso de los pinos piñoneros, que habitan en pequeñas sierras de las zonas áridas y semiáridas del norte de México, y de los pinos alpinos como $P$. hartwegii y $P$. culmi nicola. Así como de los que han evolucionado en áreas tropicales: $P$. caribea y $P$. oocarpa (Yeaton, 1982; Perry, 1991; Dvorak et al., 2000).

En México, los pinos tienen gran importancia ecológica, económica y social. A menudo son el componente dominante de la vegetación, influyen en los procesos funcionales del ecosistema tales como los ciclos biogeoquímicos, hidrológicos, los regímenes de fuego, y son hábitat y fuente de alimento para la fauna silvestre. Tienen un alto valor económico, ya que son fuente de madera, leña, pulpa, resinas, semillas comestibles y otros productos. Además, ofrecen importantes servicios ambientales (agua, oxígeno, recreación, captura de carbono) e influyen en el clima regional (García y González, 2003; Ramírez-Herrera et al., 2005).

En este trabajo, producto de una revisión bibliográfica, se describen el origen, diversidad y distribución de los pinos de México a la luz de los conocimientos actuales. También se abordan aspectos ecológicos y la problemática actual del género, con énfasis en las especies registradas en alguna categoría de riesgo.

\section{RESULTADOS Y DISCUSIÓN}

\section{Diversificación y migración de los pinos}

Se considera que durante la era Mesozoica y Cenozoica (hasta la segunda mitad del Pleistoceno), Norteamérica estuvo conectada intermitentemente con el noreste de Asia en el área que comprende el actual Mar y Estrecho de Bering. Durante este tiempo, las conexiones terrestres entre Norteamérica y Europa, a través de Groenlandia e Islandia, sirvieron de rutas migratorias para los pinos del noreste de Asia hacia Norteamérica y los del noreste de Norteamérica hacia el norte de Europa (Axelrod, 1986; Willis y McElwain, 2002).

Durante el Cretácico inferior, entre 130 y 90 millones de años, los pinos se habían diferenciado en dos subgéneros: Haploxylon y Diploxylon (actualmente Strobus y Pinus, respectivamente) y estaban ampliamente distribuidos en lo que actualmente es Canadá y Estados Unidos de América (Mirov, 1967). El cambio climático que ocurrió en el Eoceno tardío, entre 55 y 37 millones de años, provocó que las angiospermas, que estaban adaptadas a condiciones tropicales, declinaran dramáticamente en latitudes medias, ello favoreció la diversificación del género Pinus (Richardson y 
Rundel, 1998). En el Terciario tardío ocurrieron grandes levantamientos en el oeste de Norteamérica, que alteraron radicalmente su topografía y tuvieron efectos igualmente importantes sobre las poblaciones de pinos de esta vasta área. Muchas poblaciones desaparecieron por completo, mientras que otras se redujeron a relictos. Estos eventos de formación de grandes cadenas montañosas en algunas áreas, con los subsecuentes cambios climáticos, crearon la heterogeneidad ambiental que permitió la migración de Pinus hacia otras áreas que se convirtieron en centros de diversificación secundaria en México y en el noreste de Asia (Axelrod, 1986; Perry, 1991).

Durante el Pleistoceno, entre 1.7 y 0.01 millones de años las poblaciones y especies de pinos migraron hacia el sur o hacia el norte, de acuerdo con los ciclos glaciares e interglaciares. Estas fluctuaciones climáticas pudieron influir en el origen de nuevas especies o al menos afectar a la diversidad genética del género (Richardson y Rundel, 1998).

Los eventos de los últimos diez mil años, han modelado la distribución natural actual de los pinos, desde las regiones árticas y subárticas de Norteamérica y Eurasia hasta las regiones tropicales y subtropicales de Centroamérica y Asia. Su distribución más meridional en América es en Nicaragua $\left(12^{\circ} \mathrm{N}\right)$, donde se encuentran poblaciones de $P$. caribaea var. hondurensis y sólo la especie $P$. merkusii se distribuye al sur del Ecuador ( $2^{\circ} 06^{\prime}$ S) en Sumatra (Mirov, 1967; Price et al., 1998; Earle, 2007).

\section{Migración de los pinos a México}

Los primeros pinos llegaron al actual territorio mexicano en el Cretácico tardío, o en el Terciario temprano, a través de la Sierra Madre Occidental, y en el Terciario medio, desde el oriente de los Estados Unidos de América (EUA) a través de la Sierra Madre Oriental. Otra migración post-glacial provino del Este de las Montañas Rocosas por la Sierra Madre Oriental, a lo largo de las montañas y lomeríos aislados del norte de la Altiplanicie Mexicana. El avance y retroceso de los grandes glaciares favoreció la migración, muchas especies tomaron ventaja de los periodos de calor y clima seco del norte y ocuparon los espacios abiertos por el retroceso de los glaciares. La Faja Volcánica Transmexicana (FVT), sirvió como punto de contacto entre las especies de las rutas de la Sierra Madre Occidental y la Oriental, y posteriormente como un centro de diversificación del género (Eguiluz-Piedra, 1985; Perry, 1991; Rzedowski, 1998).

Varios taxa emigraron de regreso al norte por las dos sierras, donde probablemente la subsección Ponderosae y Australes tomaron la ruta al norte por la Sierra Madre Oriental hasta Nuevo León, Coahuila y Tamaulipas, y por la Sierra Madre Occidental hasta Durango, Chihuahua, Sonora y Sinaloa. Los pinos de La Rumorosa, Sierra de San Pedro Mártir y Sierra Juárez, llegaron a México a través de la Sierra Nevada, suroeste de los EUA. Algunas especies de la FVT migraron hacia el sur, rodeando la depresión del Río Balsas por el macizo montañoso del norte de Oaxaca, arribaron a las montañas de Chiapas a mediados del Terciario y de ahí migraron a Guatemala, Honduras, El Salvador y Nicaragua (Eguiluz-Piedra, 1985).

\section{Taxonomía}

México posee mayor número de especies de pinos que cualquier otra región de tamaño semejante en el mundo. Shaw (1909) registró 18 especies y 17 variedades para México; Standley 
(1920-19226) cita 28 especies y dos variedades; Martínez (1948) 39 especies, 18 variedades y nueve formas; Loock (1950) considera 55 especies de pinos para México y Honduras; Mirov (1967) 29 especies para México; Eguiluz-Piedra (1985) reconoció 69 taxa (especies, subespecies y variedades) y Perry (1991) 52 especies (14 variedades, 2 subespecies y 4 formas) para México y Centroamérica; Farjon y Styles (1997) y Perry et al. (1998) estimaron 47 especies para México y Centroamérica.

Las clasificaciones recientes del género Pinus, basadas en ADN ribosomal (Liston et al., 1999) y secuencias de ADN de cloroplastos (Krupkin et al., 1996; Gernandt et al., 2003; Zhang y Li, 2004; Gernandt et al., 2005) permiten reconocer 46 especies, 3 subespecies y 22 variedades para México. Si se incluye Pinus oocarpa var. trifoliata como una nueva especie (Pinus luzmariae), como propone Pérez de la Rosa (1998), el número de especies de pinos presentes en México sería de 47 (Tabla 1). De tal manera que al menos 26 de estas 47 especies son endémicas $(55 \%)$, sin considerar las diferentes subespecies y variedades.

\section{Distribución de pinos en México}

En Norteamérica se reconocen tres regiones de alta diversidad de especies de pinos: 1) México, 2) California, 3) sureste de EUA (Farjon y Styles, 1997). Las especies de pinos de México llegan hasta EUA, Canadá y en América Central hasta Nicaragua.

La información recopilada de diversas fuentes (ver pie de Tabla 1) indica que los pinos de México se distribuyen en cinco regiones principales, que difieren sólo parcialmente de las seis áreas que definió Eguiluz-Piedra (1985), con base en la presencia de grupos particulares de especies (Fig. 1, Tabla 1):

\section{Baja California Norte y Sur}

II. Sierra Madre Occidental: Chihuahua, Durango, Jalisco, Nayarit, Sinaloa, Sonora y Zacatecas.

III. Sierra Madre Oriental: Coahuila, Nuevo León, Querétaro, San Luis Potosí y Tamaulipas.

IV. Faja Volcánica Transmexicana: Aguascalientes, Colima, Distrito Federal, Estado de México, Guanajuato, Hidalgo, Michoacán, Morelos, Puebla, Tlaxcala y Veracruz.

V. Sierra Madre del Sur, Macizo de Oaxaca, Sierra de San Cristóbal, Sierra de Oaxaca y Península de Yucatán: Chiapas, Guerrero, Oaxaca, Campeche y Quintana Roo.

Los pinos insulares y costeros de la región I son relictos de una flora terciaria confinada a nieblas de influencia marina y tienen distribución disyunta, provocada por la separación de los desiertos de Sonora y Mojave. En esta región se reportan seis especies y siete variedades de pinos: $P$. attenuata, $P$. contorta var. murrayana, $P$. jeffreyi y $P$. lambertiana se distribuyen en las partes altas de una falla que data del Terciario y llega hasta California. Las especies $P$. coulteri, $P$. monophylla, $P$. muricata y $P$. quadrifolia, presentes en zonas de menor altitud, pudieron dispersarse desde sus centros de origen después de la desertización del suroeste de EUA (Farjon y Styles, 1997). Dos pinos insulares: $P$. radiata var. binata y $P$. cembroides ssp. lagunae son endémicos de BCN y BCS, respectivamente (Tabla 1, Fig. 1).

Las migraciones ocurridas desde las Montañas Rocosas de EUA hacia México pueden explicar, en parte, la riqueza de pinos de las regiones II y III. La región II es la de mayor diversidad, con 32 taxa 
Tabla 1. Clasificación y distribución de los pinos de México

\begin{tabular}{|c|c|c|c|c|c|c|c|}
\hline \multirow{2}{*}{ Clasificación } & \multicolumn{5}{|c|}{ Distribución por región } & \multirow{2}{*}{$\mathrm{E}$} & \multirow{2}{*}{ C } \\
\hline & 1 & II & III & IV & V & & \\
\hline \multicolumn{8}{|l|}{ Pinus subgénero Pinus } \\
\hline \multicolumn{8}{|l|}{ Sección Trifoliae } \\
\hline \multicolumn{8}{|l|}{ Subsección Contortae } \\
\hline 1. P. contorta var. murrayana (Balf.) Engelm. & 1 & 0 & 0 & 0 & 0 & & a \\
\hline \multicolumn{8}{|l|}{ Subsección Australes } \\
\hline 2. P. attenuata Lemmon & 1 & 0 & 0 & 0 & 0 & & a \\
\hline 3. P. caribaea var. hondurensis (Sénécl.) W.H.G. & 0 & 0 & 0 & 0 & 1 & & b \\
\hline 4. P. herrerae Martínez & 0 & 1 & 0 & 1 & 1 & * & \\
\hline 5a. P. greggii Engelm. ex Parl. var. australis & 0 & 0 & 1 & 1 & 0 & * & \\
\hline 5b. P. greggii Engelm. ex Parl. var. greggii & 0 & 0 & 1 & 0 & 0 & * & \\
\hline 6. P. jaliscana Pérez de la Rosa & 0 & 1 & 0 & 0 & 0 & * & a \\
\hline 7. P. lawsonii Roezl ex Gordon & 0 & 1 & 0 & 1 & 1 & * & \\
\hline 8a. P. leiophylla Schiede ex Schltdl. \& Cham. var. Ieiophylla & 0 & 1 & 1 & 1 & 1 & * & \\
\hline 8b. P. leiophylla var. chihuahuana (Engelm.) Shaw & 1 & 1 & 0 & 0 & 0 & & \\
\hline 9. P. lumholtzii B.L. Rob. \& Fernald & 0 & 1 & 0 & 1 & 0 & * & \\
\hline 10. P. muricata D. Don var. muricata & 1 & 0 & 0 & 0 & 0 & * & c \\
\hline 11a. P. oocarpa Schiede ex Schltdl. var. oocarpa & 0 & 1 & 1 & 1 & 1 & & \\
\hline 11b. P. oocarpa var. trifoliata Martínez & 0 & 1 & 0 & 1 & 1 & $\begin{array}{l}* \\
\dagger\end{array}$ & \\
\hline 12a. P. patula Schltdl. \& Cham. var. patula & 0 & 0 & 1 & 1 & 1 & * & \\
\hline 12b. P. patula var. Iongepedunculata Look ex Martínez & 0 & 0 & 0 & 0 & 1 & * & \\
\hline 13. P. praetermissa Styles \& McVaugh & 0 & 1 & 0 & 0 & 0 & * & \\
\hline 14. P. pringlei Shaw & 0 & 0 & 0 & 1 & 1 & * & \\
\hline 15. P. radiata var. binata (Engelm.) Lem mon & 1 & 0 & 0 & 0 & 0 & * & $\underset{\#}{a}$ \\
\hline 16. P. tecunumanii Eguiluz \& J.P. Perry & 0 & 0 & 0 & 0 & 1 & * & \\
\hline 17. P. teocote Schltdl. \& Cham. & 0 & 1 & 1 & 1 & 1 & * & \\
\hline \multicolumn{8}{|l|}{ Subsección Ponderosae } \\
\hline 18a. P. arizonica var. cooperi (C.E. Blanco) Farjon & 0 & 1 & 0 & 0 & 0 & * & \\
\hline 18b. $P$ arizonica var. arizonica (Engelm.) Shaw & 1 & 1 & 1 & 0 & 0 & & \\
\hline 18c. P. arizonica var. stormiae Martínez & 1 & 0 & 1 & 0 & 0 & & \\
\hline 19. P. coulteri Lamb. ex D. Don & 1 & 0 & 0 & 0 & 0 & * & a \\
\hline 20. P. devoniana Lindl. & 0 & 1 & 1 & 1 & 1 & & \\
\hline 21. P. douglasiana Martínez & 0 & 1 & 0 & 1 & 1 & * & \\
\hline 22. P. durangensis Martínez & 0 & 1 & 1 & 1 & 0 & * & a \\
\hline 23. P. engelmannii Carrière & 1 & 1 & 1 & 0 & 0 & * & \\
\hline 24. P. hartwegii Lindl. & 0 & 1 & 1 & 1 & 1 & & \\
\hline 25. P. jeffreyi Balf. & 1 & 0 & 0 & 0 & 0 & & a \\
\hline 26. P. maximinoi H.E. Moore & 0 & 1 & 0 & 1 & 1 & & \\
\hline 27. P. montezumae Lamb. var. montezumae & 0 & 1 & 1 & 1 & 1 & & \\
\hline 28. P. ponderosa var. scopulorum Engelm. & 1 & 1 & 0 & 0 & 0 & & \\
\hline
\end{tabular}


concluye Tabla 1

\begin{tabular}{|c|c|c|c|c|c|c|c|}
\hline \multirow{2}{*}{ Clasificación } & \multicolumn{5}{|c|}{ Distribución por Región } & \multirow{2}{*}{$\mathrm{E}$} & \multirow{2}{*}{$\mathrm{C}$} \\
\hline & I & II & III & IV & V & & \\
\hline \multicolumn{8}{|l|}{ Subsección Ponderosae } \\
\hline 29a. P. pseudostrobus Lindl. var. pseudostrobus & 0 & 1 & 1 & 1 & 1 & & \\
\hline 29b. P. pseudostrobus var. apulcensis (Lindl.) Martínez & 0 & 0 & 1 & 1 & 1 & * & \\
\hline \multicolumn{8}{|l|}{ Pinus subgénero Strobus } \\
\hline \multicolumn{8}{|l|}{ Sección Quinquefoliae } \\
\hline \multicolumn{8}{|l|}{ Subsección Strobus } \\
\hline 30a. P. ayacahuite var. ayacahuite & 0 & 0 & 0 & 1 & 1 & & \\
\hline 30b. P. ayacahuite var. veitchii (Roezl) Shaw & 0 & 1 & 1 & 1 & 1 & & \\
\hline 31. P. lambertiana Douglas & 1 & 0 & 0 & 0 & 0 & * & \\
\hline 32. P. flexilis var. reflexa Engelm. & 1 & 1 & 1 & 0 & 0 & * & a \\
\hline 33. P. strobiformis Engelm. * & 1 & 1 & 1 & 1 & 0 & * & \\
\hline 34. P. strobus var. chiapensis Martínez & 0 & 0 & 0 & 1 & 1 & & a \\
\hline \multicolumn{8}{|l|}{ Sección Parrya } \\
\hline \multicolumn{8}{|l|}{ Subsección Cembroides } \\
\hline 35a. P. cembroides Zucc. ssp. cembroides & 1 & 1 & 1 & 1 & 1 & & \\
\hline 35b. P. cembroides ssp. lagunae (Rob.-Pass.) D.K. Bailey & 1 & 0 & 0 & 0 & 0 & * & a \\
\hline 35c. P. cembroides ssp. orizabensis D.K. Bailey & 0 & 0 & 0 & 1 & 0 & & \\
\hline 36. P. culminicola Andresen \& Beaman & 0 & 1 & 1 & 0 & 0 & * & a \\
\hline 37. P. discolor D.K. Bailey \& Hawksw. & 1 & 1 & 1 & 0 & 0 & * & a \\
\hline 38. P. edulis Engelm. & 1 & 1 & 0 & 0 & 0 & & \\
\hline 39. P. johannis Rob.-Pass. & 0 & 1 & 1 & 0 & 0 & & \\
\hline 40. P. maximartinezii Rzed. & 0 & 1 & 0 & 0 & 0 & * & $\mathrm{C}$ \\
\hline 41. P. monophylla Torr. \& Frém. & 1 & 0 & 0 & 0 & 0 & * & a \\
\hline 42. P. pinceana Gordon & 0 & 1 & 1 & 1 & 0 & * & a \\
\hline 43. P. quadrifolia Parl. ex Sudw. & 1 & 0 & 1 & 0 & 0 & * & a \\
\hline 44. P. remota (Little) D.K. Bailey \& Hawksw. & 1 & 1 & 1 & 0 & 0 & & a \\
\hline 45. P. rzedowskii Madrigal \& Caballer o & 0 & 0 & 0 & 1 & 0 & * & a \\
\hline \multicolumn{8}{|l|}{ Subsección Nelsoniae } \\
\hline 46. P. ne/sonii Shaw & 0 & 0 & 1 & 1 & 0 & * & a \\
\hline
\end{tabular}

Clasificación de taxa basada en Liston et al. (1999), Ramírez-Herrera et al. (2005) y Gernandt et al. (2005). Distribución de taxa basada en diversas fuentes: artículos publicados en Acta Botánica Mexicana, Anales del Insti tuto de Biología de la UNAM (Serie Botánica), Boletín de la Sociedad Botánica de México, Ciencia UANL, Polibotánica, Revista Mexicana de Biodiversidad, Listados Florísticos de México del Instituto de Biología de la UNAM, Páginas Web de Jardín Botánico de Missouri y del Jardín Botánico de New York; Perry (1991), Farjon et al. (1997), Farjon y Styles (1997), Perry et al. (1998), Richardson y Rundel (1998), García y González (2003), Ramírez-Herrera et al. (2005), Earle (2007).

* Taxa endémicos (E) en México; categorías de riesgo (C) según la Norma Oficial Mexicana-2001: Protección especial (a); Amenazada (b), Peligro de extinción (c); † Propuesta como especie nueva por Pérez de la Rosa (1998); \# Según la Unión Internacional para la Conservación de la Naturaleza (UICN). 


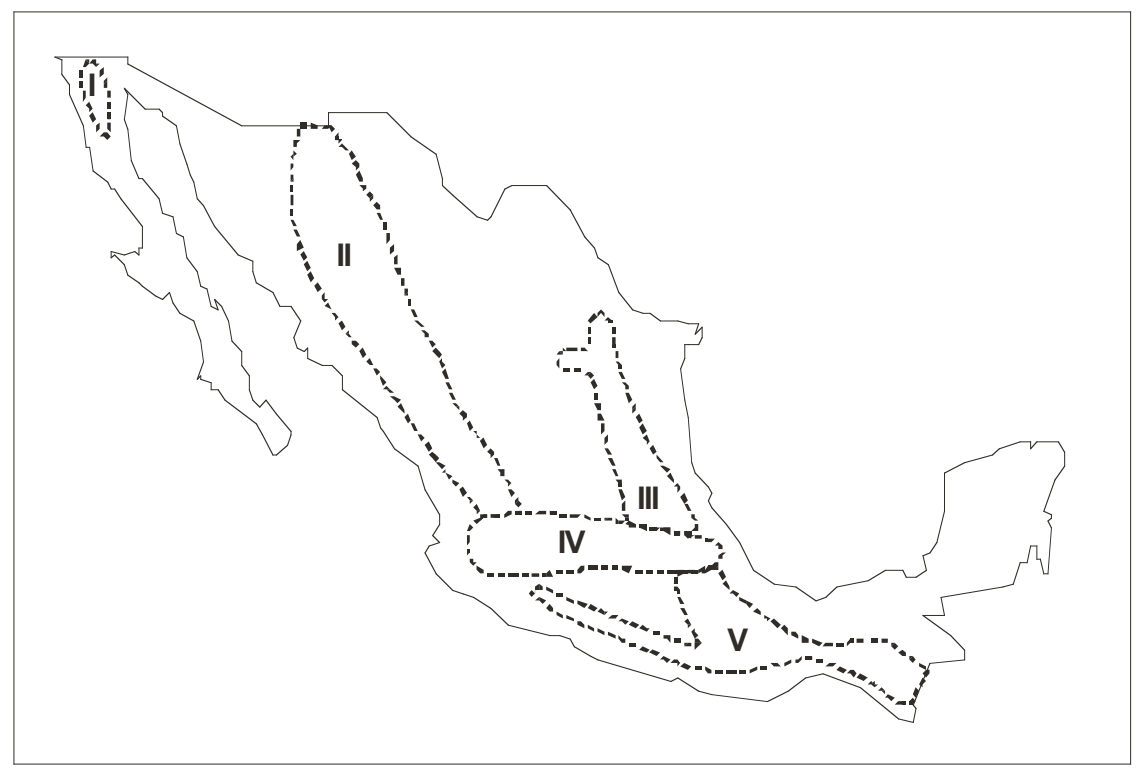

Figura 1. Distribución de los taxa de pinos en México. Modificado de Eguiluz-Piedra (1985), quien originalmente dividió la región $V$ en dos áreas diferentes.

(Fig. 2), cuatro de los cuales son endémicos: $P$. praetermissa, $P$. arizonica var. cooperi, Pinus jaliscana y P. maximartinezii (Tabla 1). Los taxa presentes en México y EUA: $P$. flexilis var. reflexa y $P$. ponderosa var. scopu lorum, están estrechamente relacionados con los taxa restringidos al norte de México: $P$. arizonica (con tres variedades), $P$. duran gensis, $P$. engelmannii, $P$. flexilis var. reflexa y $P$. strobiformis, lo que sugiere un origen común. La baja diversificación de los pinos piñoneros de ambas naciones $(P$. cembroides ssp. cembroides, $P$. discolor, $P$. edulis y $P$. remota) y su coevolución con especies de córvidos (para la dispersión de semillas) parece indicar una radiación relativamente reciente, influida por el cambio de clima (Axelrod, 1986; Farjon y Styles, 1997; Gernandt et al., 2003).

En la región III (noreste de México), la riqueza de pinos es de 25 taxa, las especies $P$. culminicola y $P$. pinceana prácticamente restringidas a esta región, la señalan como el probable centro de origen de las especies de la Subsección Cembroides (Farjon y Styles, 1997).

En la región IV (FVT) están presente 26 taxa, dos de ellos son endémicos: $P$. rzedowski y $P$. cembroides ssp. orizabensis (Tabla 1, Fig. 1, 2). Los endemismos locales de diversos grupos de plantas están ausentes, en general, hacia el sur de la FVT, lo cual es un indicio de la escasa especiación en el sur de México y en Centroamérica (Rzedowski, 1998). En el sureste de México (región $\mathrm{V}$ ) los pinos endémicos son: $P$. tecu numanii (Dvorak y Raymond, 1991; Dvorak et al., 2000) y $P$. patula var. longepeduncu lata, que pueden ser taxa derivados recientemente. La mayoría de los taxa del sur son de amplia distribución (71\%). El que la región IV y V compartan entre sí 19 taxa, señala a la migración como la causa más probable de la riqueza de pinos en Mesoamérica (Rzedowski, 1978; Farjon y Styles, 1997; Dvorak et al., 2000). 


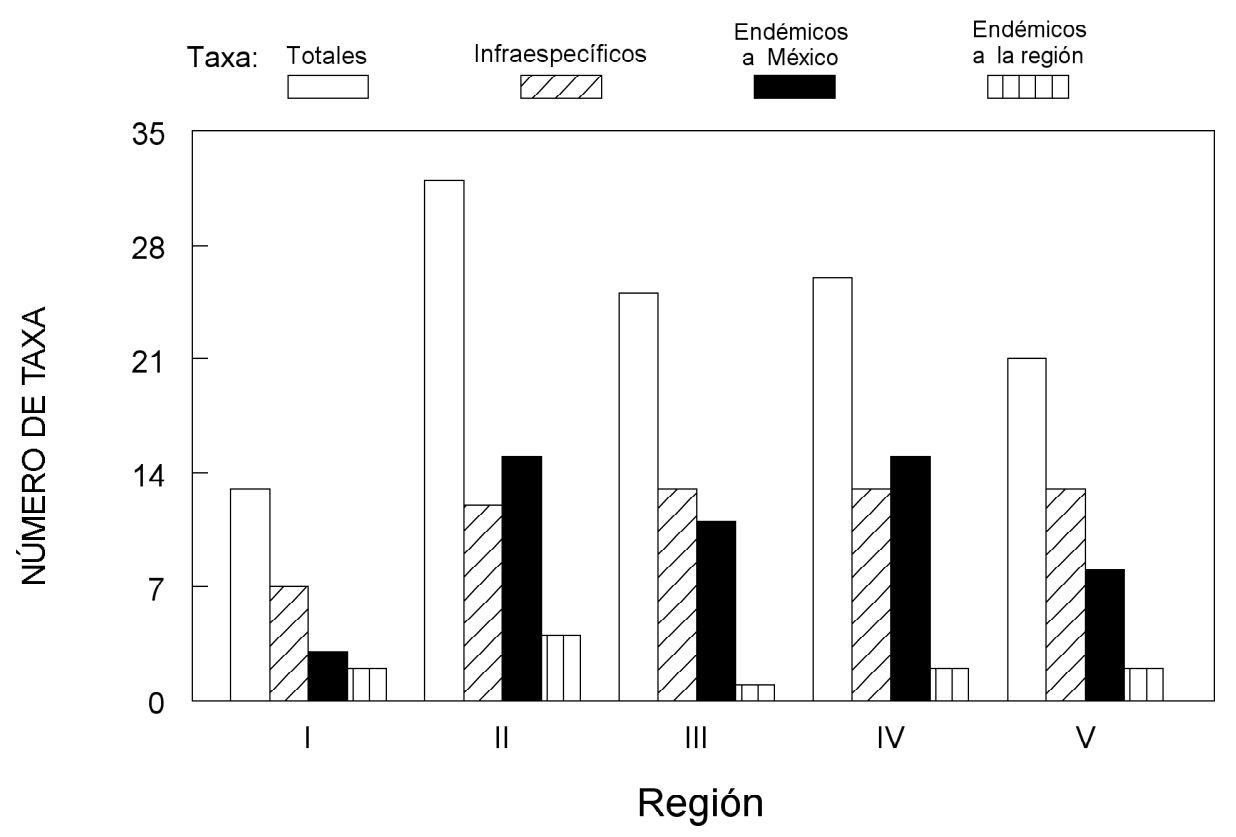

Figura 2. Riqueza y endemismos de los taxa de pinos de México por región

La diversidad disminuye drásticamente hacia el Caribe, con sólo $P$. cari baea var. hondurensis en Campeche y Quintana Roo (incluidos en la región V) y cuatro especies en Nicaragua.

Los datos precedentes, sugieren dos probables centros de diversidad y evolución de Pinus en México: El primero conformado por la FVT (Región IV), con extensiones hacia el noroeste y sureste, a través de la Sierra Madre Occidental (Región II), Sierra Madre Oriental (Región III) y Sierra Madre del Sur (región V), con las que comparte 17, 19 y 17 taxa, respectivamente. La mayoría de las entidades federativas de la República Mexicana dentro de la región IV, poseen entre 11 y 18 taxa, y sólo Aguascalientes, Colima y Guanajuato poseen menos de diez especies. El $70 \%$ de los pinos de este primer centro de diversidad son mexicanos sensu stricto y es el área de distribución principal de tres complejos de especies polimórficas: $P$. devoniana, $P$. montezumae y $P$. pseudostrobus, desde la cual pudieron dispersarse hacia otras regiones de México y Centroamérica (Farjon y Styles, 1997).

El segundo centro de diversidad (Regiones II y III), se caracteriza por los endemismos locales, originados en pequeñas montañas de las planicies áridas y semiáridas. Los eventos geológicos en el Cretácico tardío-Terciario temprano, que generaron el patrón de montañas separadas por llanuras, aunado al cambio climático relacionado con la desertización durante el Mioceno, favorecieron la migración de pinos desde las Montañas Rocosas y su diversificación posterior en esta región de México (Axelrod, 1986; Farjon y Styles, 1997). 


\section{Ecología}

Los bosques mexicanos de pino son en su mayoría resistentes a heladas, a periodos de sequía, a incendios frecuentes, al pastoreo y otros tipos de disturbio. Se pueden establecer sobre suelos someros, rocosos y muchas veces pobres en nutrientes, pero pueden colonizar sitios más favorables. Las diferencias morfológicas y fisiológicas entre las especies dominantes, la diversidad de especies acompañantes y la variedad de condiciones ecológicas en las que prosperan, son responsables de la amplia gama de variantes dentro del tipo fisonómico general de este bosque (Rzedowski, 1978).

La distribución altitudinal de los pinos varía entre 1,500 y $3,000 \mathrm{~m}$, pero pueden alcanzar el límite superior de la vegetación arbórea (3,650 m en el norte y $4,000 \mathrm{~m}$ en el centro y sur de México) (Yeaton, 1982; Earle, 2007). En los pinares la temperatura media anual fluctúa entre 6 y $28^{\circ} \mathrm{C}$ y la precipitación promedio anual de 350 a más de 1,000 $\mathrm{mm}$. Con respecto al sustrato geológico, parecen preferir los suelos derivados de rocas ígneas, pero se les puede encontrar sobre gneiss, esquistos, margas, areniscas lutitas y calizas. El color del suelo, la textura y el contenido de nutrientes, presentan variaciones considerables de un lugar a otro; son bastante frecuentes los suelos rojos, negros o muy oscuros. Algunos pinares se desarrollan en suelos con deficiencia en minerales, donde es probable que las micorrizas influyan significativamente en su supervivencia y potencial competitivo (Rzedowski, 1978).

Aunque las especies y taxa infraespecíficos endémicos están restringidos a México, algunos son de amplia distribución (Tabla 1, Fig. 2). Styles (1998) sugiere que el término endemismo se aplique sólo a los pinos con áreas de distribución limitadas o de escasa frecuencia, que habitan en condiciones ecológicas especiales. Varios taxa del subgénero Strobus, que presentan poblaciones únicas o pequeñas y disyuntas en el norte de México, pertenecen a esta categoría: $P$. cembroides ssp. cembroides, $P$. cembroides ssp. lagunae, $P$. culmini cola, $P$. discolor, $P$. edulis, $P$. johannis y $P$. remota (Tabla 1). Las especies $P$. nelsonii, $P$. pinceana y $P$. maximartinezii son más significativas: las dos primeras tienden a ser árboles aislados o forman pequeños grupos asociados con $P$. cembroides, pero no forman bosques puros o mixtos. $P$. maximartinezii sólo se ha encontrado en una localidad, en la Sierra de Morones, Zacatecas (Styles, 1998). P. rzedowskii se distribuye en tres pequeñas localidades disyuntas en Michoacán y hay poca información sobre su biología (Farjon y Styles, 1997; Delgado et al., 1999; Earle, 2007).

\section{Usos y conservación}

Los tipos de vegetación representativos de la zona templada subhúmeda de México son el bosque de pino, el bosque de pino-encino, el bosque de encino y el bosque de oyamel (Rzedowski, 1978). Dentro de estos tipos de vegetación, base de la industria forestal, $60 \%$ de las especies de pino tienen importancia comercial $y$ el $80 \%$ de los productos forestales de México se obtienen de los bosques de pino-encino (Toledo et al., 1993; SáenzRomero et al., 2003).

Los pinos son uno de los recursos naturales más valiosos para México, la madera para aserrar es el producto más importante, siguiendo la pulpa de madera para papel kraft y cartón. La importancia comercial de muchas especies de pinos "duros", se debe a que el xilema produce fibras largas que hacen que el producto final tenga gran resistencia. Los pinos 
"blandos", de menor abundancia, son requeridos por su madera menos resinosa y uniformemente granulada (Le Maitre, 1998; García y González, 2003; Ramírez-Herrera et al., 2005). En México las especies más explotadas son $P$. patula, $P$. oocarpa, P. pseudostrobus, $P$. herrerae, $P$. leiophylla y $P$. arizonica (Perry, 1991; Le Maitre, 1998; RamírezHerrera et al., 2005), pero es común que los árboles se talen sin considerar la especie.

En el ámbito local son explotados como leña, para postes, construcción de casas y muebles. En el norte de México, $P$. cembroides es fuente de leña, carbón y en menor proporción, para obtener madera. La colecta de semillas comestibles de los pinos piñoneros se practica desde tiempos precolombinos (Laner, 1981). Un producto secundario importante es la resina (se obtiene principalmente de los pinos duros), que es la materia prima para la elaboración de aguarrás y otros productos. Esta industria es fuente importante de ingresos en Chiapas, Oaxaca, Michoacán, Jalisco y Nuevo León; las especies utilizadas son Pinus oocarpa, P. montezumae, $P$. teocote y P. pseudostrobus (Styles, 1998; García y González, 2003).

Desde hace algún tiempo algunos países se han interesado en los pinos de México y Centroamérica, de manera especial en las especies subtropicales para utilizarlas en programas de reforestación. Ha sido sugerido el uso de especies como Pinus caribaea, P. oocarpa, $P$. tecunumanii, $P$. pseudostrobus, $P$. maxi minoi y $P$. chiapensis. Hay datos que confirman que la especie Pinus radiata se ha introducido en Sudamérica, Nueva Zelanda, África y Australia (Le Maitre, 1998; Earle, 2007). En Chile hay plantadas con esta especie alrededor de $1,400,000$ ha y en España 270,000 ha (Dans del Valle et al., 1999).
El área total que cubren los pinares en México es de aproximadamente $5 \%$ del territorio, valor que fue 2 o 3 veces mayor en la época prehispánica (Rzedowski, 1978). La pérdida de los bosques templados de México es de alrededor de 167,000 ha por año. La explotación forestal inadecuada, sobre todo la clandestina, los incendios y los desmontes para ampliar las zonas agrícolas, ganaderas y habitacionales, constituyen factores que restan superficie a los bosques y modifican su composición y estructura (Toledo et al., 1993; SáenzRomero et al., 2003).

En relación con la situación del género, desde el punto de vista de su conservación, existen 20 taxa de pinos incluidos en alguna categoría de riesgo dentro de la Norma Oficial Mexicana (NOM-059-ECOL-2001). Las especies de distribución restringida son las más vulnerables. García y González (1991) han registrado que las áreas ocupadas por el matorral de $P$. culminicola en el Cerro Potosí, Nuevo León, se han reducido considerablemente; la causa principal es el fuego, pues no es una especie que lo resista (Tabla 1 ).

$P$. rzedowskii se distribuye en Michoacán, en un área de aproximadamente 1.6 millones de ha, donde se encuentran doce pequeñas poblaciones fragmentadas, algunas de ellas con densidades muy bajas, por lo que sorprende que la especie posea niveles de variación genética comparables a los de otras especies de coníferas con poblaciones grandes (Delgado et al., 1999; Earle, 2007).

En el caso de $P$. maximartinezii, se encuentra confinado al sur del estado de Zacatecas, donde existe una población de entre 2,000 y 2,500 árboles maduros, que cubren alrededor de 400 ha. El bosque es propiedad privada, usada para 
pastoreo y para la recolección de piñón. Aunque existe preocupación por esta especie, es posible que el manejo adecuado del bosque por los habitantes de la zona pueda ayudar a su conservación in situ (Styles, 1998; Ledig et al., 2001). P. rzedowskii y P. maximartinezii, son dos de las especies más raras y amenazadas (Perry, 1991; NOM, 2001; Earle, 2007).

$P$. nelsonii es de distribución más restringida que $P$. pinceana, pero se encuentra en sitios menos explotados por el hombre. El principal peligro para $P$. pinceana es el pastoreo (Ledig et al., 2001). Por otro lado, aún cuando las poblaciones isleñas de $P$. radiata var. binata están siendo seriamente diezmadas por el ganado caprino, especialmente en Isla Guadalupe (Perry, 1991), la especie no se ha incluido en la NOM (2001). Todos los taxa vulnerables continúan sin protección, por lo que se requiere impulsar acciones para su conservación (Tabla 1).

\section{CONCLUSIONES}

En México existen 46, o tal vez 47 especies de pinos, que representan más de $42 \%$ de las especies conocidas en el mundo: esta diversidad es aún mayor si tomamos en cuenta las subespecies y variedades. La síntesis de varias disciplinas, entre ellas la sistemática molecular, confirma esta información con un bajo margen de error. Con respecto a la distribución de Pinus, sólo las zonas tropicales de baja altitud de los estados de Tabasco y Yucatán carecen de pinos nativos; otros estados como Chihuahua, Coahuila, Durango, Hidalgo, Jalisco, México, Michoacán, Nuevo León, Puebla, Veracruz y Zacatecas tienen más de 16 especies cada uno. Como muchas de las áreas de distribución de los pinos tienden a desaparecer en función del continuo crecimiento de las poblaciones humanas, es imprescindible que en los planes de desarrollo de México se considere el impacto sobre los recursos naturales: al menos 20 taxa de pinos mexicanos están en alguna categoría de riesgo. Se estima que en los bosques de pino y pino-encino existe alrededor del $24 \%$ del total de la flora de México, la mayoría especies endémicas. Estos bosques mantienen una enorme diversidad de seres vivos que representan bienes y servicios importantes para el pueblo mexicano, es prioritario evitar que se extingan en un futuro cercano.

\section{AGRADECIMIENTOS}

El autor agradece a quienes posibilitaron la aparición de esta revisión: los autores de los trabajos consultados.

\section{REFERENCIAS}

Axelrod, D. I. 1986. Cenozoic history of some western american pines Annals of Missouri Botanical Garden 73: $565-641$

Dans del Valle, J.F., F.J. Fernández y A. Romero G. 1999. Manual de selvicultura del pino radiata en Galicia. Asociación Forestal de Galicia. Universidad de Santiago de Compostela, España.

Delgado, P., D. Piñero, A. Chaos, N. Pérez-Nasser and E. R. ÁlvarezBuylla. 1999. High population differentiation and genetic variation in the endangered Mexican pine Pinus rzedowskii (Pinaceae). American Journal of Botany 86: 669-676.

Dvorak, W. S. y R. H. Raymond. 1991 The taxonomic status of closely related closed cone pines in Mexico and Central America. New Forests 4: 291-307. 
Dvorak, W. S., A. P. Jordon, G. P. Hodge y J. L. Romero. 2000. Assessing evolutionary relationships of pines in the Oocarpae and Australes subsections using RAPD Markers. New Forests 20: 163-192.

Earle, J. C. 2007. Gymnosperm Database. http://www.conifers.org/index. htm. Fecha de actualización 13 de julio de 2006.

Eguiluz-Piedra, T. 1985. Origen y evolución del género Pinus (con referencia especial a los pinos mexicanos). Dasonomía Mexicana 6: 531.

Farjon, A., J. A. Pérez de la Rosa and T. B. Styles. 1997. Guía de campo de los pinos de México y América Central. Royal Botanic Gardens, Kew- University of Oxford. $151 \mathrm{p}$.

Farjon, A. y T. B. Styles. 1997. Pinus (PINACEAE). Flora Neotropica, Monograph 75. Organization for Flora Neotropica. The New York Botanical Garden, New York, USA. 291 p.

Farjon, A. 2003. The remaining diversity of conifers. Acta Horticultura (ISHS) 615: 75-89.

García, A. A. y E. S. González. 1991. Flora y vegetación de la cima del Cerro Potosí, Nuevo León, México. Acta Botánica Mexicana 13:53-74.

García A., A. y M. S. González. 2003. Pináceas de Durango. Instituto de Ecología, A.C. Comisión Nacional Forestal. México. 187 p.

Gernandt, D., A. Liston y D. Piñero. 2003. Phylogenetics of Pinus Subsections Cembroides and Nelsoniae inferred from cPDNA secuences. Systematic Botany 28: 657-673.
Gernandt, D., L. G. Geada, G. S. Ortiz y A. Liston. 2005. Phylogeny and classification of Pinus. Taxon 54: 29-42.

Hilton, J., S. J. Wang, B. Tian y C. H. Li. 2003. Evidence for conifer origins and early evolution from the late Palaeozoic Cathaysian flora of south east Asia. Acta Horticultura (ISHS) 615: 59-65.

Krupkin, A. B., A. Liston y S. H. Strauss. 1996. Phylogenetic analysis of the hard pines (Pinus subgenus Pinus, Pinaceae) from chloroplast DNA restriction site analysis. American Journal of Botany 83: 489-498.

Laner, R. M. 1981. The Pinon Pine: A Natural and Cultural History. University of Nevada Press, USA. 208 p.

Ledig, F. T., M. A. Capó-Arteaga, P. D. Hodgskiss, H. Sbay, C. FloresLópez, M. Thompson y B. BermejoVelázquez. 2001. Genetic diversity and the mating system of a rare Mexican piñon, Pinus pinceana, and a comparison with Pinus maximarti nezii (Pinaceae). American Journal of Botany 88: 1977-1987.

Le Maitre, D. C. 1998. Pines in cultivation: a global view. In: M. D. Richardson (ed). Ecology and Biogeography of Pinus. Cambridge University Press.Cambridge, UK. pp: 407-431.

Liston, A., W. A. Robinson, W. D. Piñero y E. R. Álvarez-Buylla. 1999. Phylogenetics of Pinus (Pinaceae) based on nuclear ribosomal DNA internal transcribed spacer region secuences. Molecular Phylogenetics and Evolution: 11: 95-109.

Loock E., E. M. 1950. The pines of Mexico and British Honduras. South Africa Department of Forestry Bulletin 35. South Africa. $262 \mathrm{p}$ 
Martínez, M. 1948. Los pinos mexicanos. Ediciones Botas, México. 366 p.

Miller, C. N. Jr. 1999. Implications of fossil conifers for the phylogenetics relationships of living families. The Botanical Review 65:240-277.

Mirov, N. T. 1967. The genus Pinus. Ronald Press. New York, USA. 602 p.

Norma Oficial Mexicana (2001) NOM-059ECOL-2001, Protección ambientalespecies nativas de México de flora y fauna silvestres -categorías de riesgo y especificaciones para su inclusión, exclusión o cambio- lista de especies en riesgo.

Pérez de la Rosa, J. A. 1998. Promoción de una variedad de pino serotino mexicano a nivel de especie. Boletín del Instituto de Botánica. Universidad de Guadalajara (IBUG) 5:127-135.

Perry, J. P. Jr. 1991. The pines of México and Central America. Timber Press. Portland, Oregon, USA. 231 p.

Perry, J. P. Jr., A. Graham y M. D. Richardson. 1998. The history of pines in México and Central America. In: M. D. Richardson (ed). Ecology and Biogeography of Pinus. Cambridge University Press. Cambridge, UK. pp: 137-149.

Price, R. A., A. Liston y S. H. Strauss. 1998. Phylogeny and systematics of Pinus. In: M. D. Richardson (ed). Ecology and Biogeography of Pinus. Cambridge University Press. Cambridge, UK. pp: 49-68.

Ramírez-Herrera, C., J.J. VargasHernández y J. López-Upton. 2005. Distribución y conservación de las poblaciones naturales de Pinus greggii. Acta Botánica Mexicana 72: 1-16.
Richardson, M. D. y W. P. Rundel. 1998. Ecology and biogeography of Pinus: an introduction. In: M. D. Richardson (ed). Ecology and Biogeography of Pinus. Cambridge University Press. Cambridge, UK. pp: 3-46.

Rzedowski, J. 1978. Vegetación de México. Editorial Limusa, México, D.F. 432 p.

Rzedowski, J. 1998. Diversidad y orígenes de la flora fanerogámica de México. In: T. P. Ramamorthy, R. Bye, A. Lot y J. Fa (eds). La diversidad biológica de México, orígenes y distribución, Instituto de Biología, UNAM, México. pp. 129-145.

Sáenz-Romero, C., A. E. Snively y R. Lindig-Cisneros. 2003. Conservation and restoration of pine forest genetic resources in Mexico. Silvae Genetica 52: 5-6.

SEMARNAT. 2001. Programa Nacional Forestal 2001-2006. Secretaría del Medio Ambiente y Recursos Naturales. Comisión Nacional Forestal. México. $150 \mathrm{p}$.

Shaw, G. R. 1909. The Pines of Mexico. Publications of the Arnold Arboretum No. 1.30 p.

Standley, P. C. 1920-1926. Trees and Shrubs of Mexico. Contributions from the United States National Herbarium 23: 1-1721.

Styles, T. B. 1998. El género Pinus: su panorama en México. In: T. P. Ramamorthy, R. Bye, A. Lot y J. Fa (eds). La diversidad biológica de México, orígenes y distribución. Instituto de Biología, UNAM, México. pp. 385-408.

Toledo, V. M., J. Carabias, C. Toledo y C. González-Pacheco. 1993. La producción rural en México: alterna- 
tivas ecológicas. Fundación Universo Veintiuno y Prensas de Ciencias. México. 402 p.

Willis, K. J. y J. C. McElwain. 2002. The evolution of plants. Oxford University Press. New York, USA. 378 p.

Yeaton, R. I. 1982. The altitudinal distribution of the genus Pinus in the western United States and Mexico. Boletín de la Sociedad Botánica de México 42:55-71.

Zhang, Z. Y. y D. Z. Li. 2004. Molecular phylogeny of Section Parrya of Pinus (Pinaceae) based on chloroplast matK gene sequence data. Acta Botanica Sinica 46: 171-179. 